\title{
PRE-SELF-INJECTIVE DUO RINGS
}

\author{
ANNE KOEHLER
}

\begin{abstract}
In this paper duo rings (every one-sided ideal is two sided) which are pre-self-injective (every proper homomorphic image is self-injective) are characterized. It will be shown that, for duo rings, a ring $R$ is pre-self-injective if and only if it is one of the following rings: (1) a direct sum of a finite number of rank 0 maximal valuation rings; (2) a local ring whose maximal ideal $M$ has composition length 2 and $M^{2}=0$; or (3) a domain $R$ in which every proper ideal is contained in only finitely many maximal ideals and which has, for each maximal ideal $M$ and ideal $A \subseteq M, R_{M} / A_{M}$ being a rank 0 duo maximal valuation ring for the localization at $M$.

The object of the paper is to extend the results of Klatt and Levy for commutative pre-self-injective rings [6]. Klatt and Levy's characterization has already been extended [7] to self-injective rings for which all the proper cyclic modules are quasi-injective (called qc-rings). The qc-rings are direct sums of semisimple Artinian rings and duo rings. Jain, Singh, and Symonds have also studied the noncommutative problem by looking at rings with all the cyclic modules, which are not isomorphic to the ring, being quasi-injective (called PCQI-rings). The rings in the present paper do have the property that they are PCQI-rings. Hence, use can be made of the results in [5]. However, Jain, Singh, and Symonds left unresolved how to characterize prime PCQI-rings.
\end{abstract}

1. Preliminary definitions and propositions. Every ring is assumed to have an identity. A ring is a right (left) valuation ring if for every pair of elements $x$ and $y$ in the ring, either $x \in y R$ or $y \in x R(x \in R y$ or $y \in R x)$. A valuation ring means both a left and a right valuation ring. A right valuation ring is called maximal if every family of pairwise solvable congruences of the form $x \equiv x_{\alpha}\left(\bmod J_{\alpha}\right)$, where $x_{\alpha} \in R$ and each $J_{\alpha}$ is an ideal of $R$, has a simultaneous solution (see [6]). A right valuation ring is almost maximal if all its proper homomorphic images are maximal. A ring has rank 0 (rank 1 ) if every (proper) prime ideal is a maximal ideal.

PROPOSITION 1.1. If $R$ is an almost maximal right valuation ring which is not prime, then $R$ is maximal.

Proof. Gill has proved this result for commutative rings [3]. The same proof works for noncommutative rings.

For a definition of the (right) localization $R_{P}$ of a duo ring $R$ at a prime ideal $P$, we will use the one given by Shores [9]. Brungs [2] has shown that such a

Presented to the Society, August 19, 1975; received by the editors August 13, 1975 and, in revised form, January 19, 1976.

AMS (MOS) subject classifications (1970). Primary 16A52.

Key words and phrases. Duo, self-injective, maximal valuation ring, quasi-injective.

Copyright $\odot 1977$, American Mathematical Society 
localization $R_{P}$ might not be a duo ring. The ideal generated by the image of $A$ in $R_{P}$ will be denoted by $A_{P}$. Note that Brungs' example says the right ideal generated by $A$ might be smaller than $A_{P}$.

Proposition 1.2. Let $A$ be an ideal of a duo ring $R$ which is contained in the prime ideal $P$, and let $\sigma$ be the natural ring homomorphism from $R$ onto $R / A$. Then $R_{P} / A_{P} \cong \sigma(R)_{\sigma(P)}$.

Proof. The proof is essentially the same as that of 6.2 and 6.3 in [8].

For a duo domain $R, R_{P}=\left\{a b^{-1}: a, b \in R, b \notin P\right\}$ and $a b^{-1}=c d^{-1}$ if $a d=c e$ where $b d=d e$.

Proposition 1.3. If $R$ is a duo domain, then $R_{P}$ is a domain.

Proof. Straightforward by using $a b^{-1} c d^{-1}=a e(d b)^{-1}$ where $c b=b e$.

\section{Main results.}

Proposition 2.1. A right pre-self-injective duo ring $R$ is either semiperfect or a domain.

Proof. As has been noted, $R$ is a right PCQI-ring. Hence, $R$ is either semiperfect or prime by Proposition 3 in [5]. Since $R$ is a duo ring, it is prime if and only if it is a domain.

Proposition 2.2. Suppose $R$ is a semiperfect ring which is not a domain. Then $R$ is a right pre-self-injective duo ring if and only if it is (1) a finite direct sum of rank 0 duo maximal valuation rings; or, (2) a local duo ring whose maximal ideal $M$ has composition length 2 and $M^{2}=0$.

Proof. If $R$ is semiperfect and not local, then being a pre-self-injective duo ring is equivalent to being (1) because summands of $R$ are local qc-rings. Thus, $R$ is a qc-ring and the equivalence is the Main Theorem in [7].

Now consider a local ring $R$ which is not a domain. The ring $R$ is a right pre-self-injective duo ring if and only if it is (2) or it is an almost maximal duo valuation ring of rank 0 by Theorems 14, 15, and 16 in [5]. From Proposition 1.1, when $R$ is an almost maximal rank 0 valuation ring which is not prime, it is maximal, and hence it is (1).

Proposition 2.3. $R$ is a right pre-self-injective local duo domain if and only if $R$ is an almost maximal valuation duo domain of rank 1 .

Proof. By Theorem 14 in [5], $R$ is a valuation ring. For any ideal $A, R / A$ is a local qc-ring which is equivalent to being a maximal valuation ring of rank 0 by $\$ 2$ in [7].

Proposition 2.4. Let $R$ be a duo domain. Then $R$ is right pre-self-injective if and only if

(1) every proper ideal is contained in only finitely many maximal ideals, and

(2) for every maximal ideal $M$ and every proper ideal $A_{M}$ in the localization $R_{M}, R_{M} / A_{M}$ is a maximal rank 0 duo caluation ring. 
In order to prove Proposition 2.4, we need three lemmas which will be presented next.

LEMMA 2.5. If a duo ring has only a finite number, $n$, of maximal ideals and every prime is maximal, then $R$ is the direct sum of $n$ local rings.

Proof. We will use mathematical induction on the number of maximal ideals in the ring.

If a duo ring has one maximal ideal $M$, then $R$ is a local ring. Now assume that every duo ring, in which there are $k$ maximal ideals with $k<n$ and in which all primes are maximal, is the direct sum of $k$ local rings.

Let $R$ be a duo ring with exactly $n$ maximal ideals and all primes maximal. Let $M_{1}, M_{2}, \ldots, M_{n}$ be the maximal ideals of $R$. Since $I_{i=2}^{n} M_{i} \subseteq \cap_{i=2}^{n} M_{i}$, and maximal ideals are prime, $\bigcap_{i=2}^{n} M_{i} \nsubseteq M_{1}$. Hence $R=M_{1}+\cap_{i=2}^{n} M_{i}$. Let $N=\cap_{i=1}^{n} M_{i}$. Then $R / N=\left(M_{1} / N\right) \oplus\left(\cap_{i=2}^{n} M_{i} / N\right)$ is a direct sum and $\overline{1}=\bar{a}+b$ where $\bar{a}$ and $\bar{b}$ are orthogonal idempotents in $R / N$.

Since $N$ is the prime radical, it is nil. The ideal $N$ is also the Jacobson radical. Hence we can lift the idempotents $\bar{a}$ and $\bar{b}$ to orthogonal idempotents $e$ and $f$ so that $1=e+f, \bar{a}=\bar{e}$, and $\bar{b}=\bar{f}$. Thus we have $R=\operatorname{Re} \oplus \operatorname{Rf}$ is a direct sum, and we can apply the induction hypothesis to finish the proof.

LEMma 2.6. If a duo ring is the direct sum of local rings, then the local rings are precisely the localizations of $R$ at its maximal ideals.

Proof. Let $R=A_{1} \oplus A_{2} \oplus \cdots \oplus A_{n}$ where $A_{i}, i=1, \ldots, n$, is a local ring. Let $P_{i}$ be the unique maximal ideal in $A_{i}$. Let $B_{i}=\sum_{j \neq i} \oplus A_{j}$ and $\sigma_{i}$ : $R \rightarrow R / B_{i}$ be the natural ring homomorphism. The maximal ideals of $R$ are $M_{i}=P_{i} \oplus B_{i}, i=1, \ldots, n$. Thus, by using Proposition 1.2 and $\left(B_{i}\right)_{M_{i}} \cong 0$,

$$
A_{i} \cong\left(A_{i}\right)_{P_{i}} \cong \sigma_{i}(R)_{\sigma_{i}\left(M_{i}\right)} \cong R_{M_{i}} /\left(B_{i}\right)_{M_{i}} \cong R_{M_{i}} .
$$

Lemma 2.7. If $R$ is a duo domain and if, for each maximal ideal $M$, the localization $R_{M}$ has rank 1 , then $R$ has rank 1 .

Proof. Let $P$ be a proper prime ideal of $R$ and $M$ be a maximal ideal containing $P$. In $R_{M}, b^{-1} p=c b^{-1}$ where $p b=b c$. Hence, if $p \in P$, then $c \in P$. Combining this fact with the usual definition for adding right fractions, we see $P_{M}=P R_{M}$. Now, if there is an $m \notin M-P$, one can see $m \notin P_{M}$ and $P_{M} \neq M R_{M}$. On the other hand, $R / P$ is a domain. So, if $\sigma$ : $R \rightarrow R / P, \sigma(R)_{\sigma(M)}$ is a domain by Proposition 1.3 and $\sigma(R)_{\sigma(M)} \cong R_{M} / P_{M}$ by Proposition 1.2. But, if $P_{M}$ is prime it must be maximal and equal to $M R_{M}$. Since we have a contradiction, $P=M$.

Proof of Proposition 2.4. "Only if." Assume $R$ is a duo domain which is right pre-self-injective. Let $A$ be a proper ideal of $R$. Then $R / A$ is a qc-ring and hence semiperfect by [1]. So $A$ is contained in only finitely many maximal ideals.

Let $M$ be a maximal ideal and $A$ any proper ideal contained in $M$. By Proposition $1.2, R_{M} / A_{M} \cong \sigma(R)_{\sigma(M)}$ where $\sigma: R \rightarrow R / A$. Since $R / A$ is 
semiperfect it is a direct sum of local rings, each of which is a local qc-ring. By $\S 2$ in [7], each of these local rings is a rank 0 maximal duo valuation ring. By Lemma 2.6, $\sigma(R)_{\sigma(M)}$ is isomorphic to one of these local direct summands of $R / A$.

"If." Assume $R$ is a duo domain which satisfies (1) and (2). Let $A$ be a proper ideal in $R$. Then $R / A$ has only a finite number $n$ of maximal ideals. From Lemma 2.7 every proper prime in $R$ is maximal. Hence, in $R / A$ every prime is maximal, and $R / A$ is a direct sum of $n$ local rings by Lemma 2.5. Using Lemma 2.6, we see that each of these $n$ local rings is isomorphic to a localization of $R / A$. Then by Proposition 1.2 each of these localizations is isomorphic to $R_{M} / A_{M}$ for some maximal ideal $M$. Each $R_{M} / A_{M}$ is self-injective [7]. So, each of the $n$ local rings is right self-injective. Therefore, $R / A$ is right self-injective.

REMARKS. 1. The characterization of right pre-self-injective duo rings shows that being a right pre-self-injective duo ring is equivalent to being a left pre-self-injective duo ring.

2. Notice that we have shown neither that $R_{M}$ is a duo domain, nor that it is a right PCQI-ring. If $R_{M}$ had either property, it would be a right valuation domain by Theorem 17 in [5].

ACKNOWLEDGEMENT. I wish to thank the referee for pointing out the existence of Gill's result for commutative rings and for suggesting a way to make the proof of Lemma 2.5 compact.

\section{REFERENCES}

1. Javed Ahsan, Rings all of whose cyclic modules are quasi-injective, Proc. London Math. Soc. (3) 27 (1973), 425-439. MR 49 \#2841.

2. H. H. Brungs, Three questions on duo rings, Pacific J. Math. 58 (1975), 345-349.

3. D. T. Gill, Almost maximal valuation rings, J. London Math. Soc. (2) 4 (1971), 140-146. MR 45 \# 1904.

4. N. Jacobson, Structure of rings, Amer. Math. Soc. Colloq. Publ., vol. 37, rev. ed., Amer. Math. Soc., Providence, R. I., 1964. MR 36 \#158.

5. S. K. Jain, Surjeet Singh and R. C. Symonds, Rings whose proper cyclic modules are quasi-injective (preprint).

6. G. B. Klatt and L. S. Levy, Pre-self-injective rings, Trans. Amer. Math. Soc. 137 (1969), 407-419. MR 38 \#4463.

7. A. Koehler, Rings with quasi-injective cyclic modules, Quart. J. Math. Oxford Ser. (2) 25 (1974), 51-55.

8. M. Nagata, Local rings, Interscience, New York, 1962. MR 27 \#5790.

9. Thomas S. Shores, Injective modules over duo rings, Pacific J. Math. 40 (1972), 695-702. MR 46 \#9114.

School of Business Administration, Miami University, Oxford, Ohio 45056 